\title{
Update on genetic evidence for rupture-prone compared with rupture-resistant intracranial saccular aneurysms
}

\author{
Vini G. Khurana, M.D., Ph.D., Irene Meissner, M.D., And Fredric B. Meyer, M.D. \\ Departments of Neurologic Surgery and Neurology, and the Mayo Stroke Center, Mayo Clinic and \\ Mayo Foundation, Rochester, Minnesota
}

\begin{abstract}
Object. Anecdotal evidence exists for at least two subpopulations of intracranial saccular aneurysms; those that form rapidly and rupture when small and those that enlarge slowly and are particularly prone to rupture when they are 10 $\mathrm{mm}$ or more in diameter. The goal in this study was to determine if there was genetic evidence to support the classification of intracranial saccular aneurysms as "rupture-prone" or "rupture-resistant" lesions.

Methods. The authors prospectively obtained and analyzed clinical and genetic data in a cohort of 197 individuals composed of 58 patients with ruptured intracranial saccular aneurysms, 49 with unruptured aneurysms, and 90 healthy community volunteers. Based on recent studies supporting an increasingly relevant role for the critical vasomodulatory protein endothelial nitric oxide synthase (eNOS) in aneurysm pathobiology, the authors assayed blood from all 197 participants to determine and compare their eNOS genotypes.

The eNOS gene intron 4 27-base pair variable-number tandem-repeat polymorphism was significantly overrepresented in persons with ruptured intracranial saccular aneurysms compared with community volunteers $(\mathrm{p}<0.002)$. When comparing eNOS genotypes among patients with ruptured or unruptured aneurysms, an approximately 10-fold increase in the odds of presenting with brain aneurysm rupture was found among individuals with multiple variant eNOS alleles $(\mathrm{p}=0.004)$.

Conclusions. Uniquely, the authors have identified a set of $e N O S$ gene variations whose presence indicates patients with intracranial saccular aneurysms that are more prone to rupture. The authors conclude that if these findings are reproducible in the setting of a large multicenter study, then in addition to known anatomical factors, a rapid and costeffective genetic screening tool will become available to clinicians as an aid to predicting rupture risks in patients presenting with unruptured intracranial aneurysms.
\end{abstract}

\section{KEY WORDS • functional genomics • genetic polymorphism • intracranial aneurysm • nitric oxide synthase $\bullet$ subarachnoid hemorrhage}

Our goal in this article is to provide an update on the evolving evidence for the existence of two distinct subpopulations of intracranial saccular aneurysms. One is composed of aneurysms that develop relatively rapidly and rupture when smaller than $10 \mathrm{~mm}$ in diameter (that is, the variety that tends to be seen in the emergency room setting). The other is composed of aneurysms that enlarge slowly, are amenable to study over months or years with serial imaging, and are more prone to rupture when 10 $\mathrm{mm}$ or larger in diameter (that is, those seen and followed in the clinical setting). The data summarized in this article are reported and discussed in detail elsewhere..$^{5,10,15,16}$

\footnotetext{
Abbreviations used in this paper: eNOS = endothelial nitric oxide synthase; ISUIA = International Study of Unruptured Intracranial Aneurysms; SAH = subarachnoid hemorrhage; SNP = single-nucleotide polymorphism; VNTR = variable-number tandemrepeat.
}

\section{OVERVIEW}

\section{Controversy Over Aneurysm Size in Relation to Rupture Risk}

Although there is considerable epidemiological evidence supporting a multifactorial origin for intracranial aneurysm formation, ${ }^{29}$ including factors regarded as congenital (heritable connective tissue disorders, familial predisposition, and female sex) and acquired (cigarette smoking and hypertension), there is much controversy surrounding the natural history of unruptured intracranial aneurysms. Certainly, the discrepancy between the prevalence of brain aneurysms (as high as 5\% or 10-15 million in the US population alone) and the incidence of rupture $(\sim 30,000$ cases annually in the US) indicates that some brain aneurysms are more prone to rupture than others. ${ }^{9,29}$

Particularly since the publication of the studies by Wiebers and colleagues, ${ }^{35,36}$ to improve the overall outcome in 
what is otherwise a devastating disease, a multitude of investigators have sought to identify factors that may determine which aneurysms are more prone to rupture. In a systematic and comprehensive manner, the ISUIA has shed some light on this by identifying aneurysm size of 10 $\mathrm{mm}$ or more in diameter in patients with no previous SAH and posterior location as independent predictors of rupture. ${ }^{10}$ As Forget, et al., ${ }^{5}$ have reported, however, the findings of the ISUIA are disputed by the fact that the majority of aneurysms presenting after rupture are less than 10 $\mathrm{mm}$ in size $(>85 \%$ in their report and $75 \%$ in our own series $^{16}$ ). Explanations for this discrepancy include a much higher prevalence of smaller aneurysms overall, decrease in aneurysm size after rupture, or existence of a subpopulation of aneurysms that rupture at a smaller critical size soon after formation. ${ }^{5,36}$ The former two explanations have been substantially rejected; 5 the third seems more plausible, and is the basis for the hypothesis tested in our recent genetic studies. ${ }^{15,16}$

\section{Specific Anatomical Evidence for Rupture-Prone Compared With Rupture-Resistant Aneurysms}

In our most recent study (VG Khurana, et al., 2004, manuscript in review), our groups with unruptured and ruptured aneurysms (49 and 58 patients, respectively) showed no significant difference in demographic and clinical variables such as age, sex, race, cardiovascular comorbidities, smoking, and family history of brain aneurysm or SAH. Furthermore, patient demographics and aneurysm features (such as multiplicity, mean size, and location) in our group with unruptured lesions were consistent with those reported in Group 1 patients with unruptured aneurysms in the ISUIA (that is, patients with no preceding SAH from another aneurysm). ${ }^{10}$

Aneurysms in our ruptured and unruptured groups differed significantly, however, with regard to anatomical features. Consistent with other series of ruptured brain aneurysms, $, 531,36$ anatomically, lesions in our ruptured group were smaller (mean size $7.5 \mathrm{~mm}$ ) than those in the unruptured group (mean size $9.6 \mathrm{~mm} ; \mathrm{p}=0.037$ ), and tended to be much more frequently located in the anterior and posterior communicating arteries, and much less frequently in the posterior circulation $(\mathrm{p}<0.001)$.

These findings are at odds with those of the ISUIA. ${ }^{10}$ In that study, unruptured aneurysms larger than $10 \mathrm{~mm}$ or those located in the vertebrobasilar circulation or along the posterior cerebral artery were identified as being more prone to rupture, whereas aneurysms located along the anterior communicating artery were found to rupture relatively infrequently. This disparity underscores arguments put forth by investigators that the type of lesions described in the ISUIA may in fact represent only one type of intracranial saccular aneurysm; the type that is not prone to rupture. These arguments have been articulated elsewhere. ${ }^{5,16}$

\section{Does a Genetic Approach Hold the Answer?}

In the light of mounting evidence that genetic factors may play a role in intracranial aneurysm formation and rupture, ${ }^{16,23,24,29}$ the key questions that arise are these: can genetic technologies be applied to detect different subpopulations of intracranial saccular aneurysms, and can such information be used to improve the care of patients who have these lesions? With the completion of the Human Genome Project, attention is now focused on the field of functional genomics, in which the principal aim is to establish links between genes, the proteins they encode, and the molecular pathogenesis of human disease. ${ }^{25,26}$

Why study the eNOS gene? Of the array of molecular candidates that are potentially relevant to the pathogenesis of aneurysmal SAH, we deliberately chose the gene encoding the endothelial isoform of NOS as the focus for our investigation for several reasons. First, NO derived from constitutive isoforms of NOS acts as a potent vasodilator and inhibitor of inflammation, smooth-muscle cell proliferation, and platelet aggregation. ${ }^{2,21}$ Second, there is biochemical, immunohistochemical, and functional evidence for impairment of NO signaling after experimental SAH in animals, ${ }^{12,13,34}$ and abnormal NO levels in cerebrospinal fluid have been reported in humans following aneurysmal SAH. ${ }^{28}$ Third, in gene transfer paradigms, recombinant eNOS overexpression in animal ${ }^{13}$ and human ${ }^{14}$ intracranial arteries, even in the setting of experimental SAH, has been shown to be vasoprotective. Fourth, eNOS gene knockout in animals has been shown to predispose them to hypertension, atherosclerosis, coronary artery disease, and aortic aneu-rysm formation. ${ }^{18}$ Fifth, the inducible isoform of NOS and metabolically uncoupled eNOS can generate free radicals that adversely affect the structure and function of vessel walls. ${ }^{6,21}$ Last, in several studies investigators have shown that the gene encoding eNOS, which is located on chromosome 7q35$36,{ }^{20}$ is polymorphic in humans and that the presence of certain eNOS polymorphisms may increase susceptibility to conditions including atherosclerosis, hypertension, myocardial infarction, coronary vasospasm, and formation of abdominal aortic aneurysms. . $^{7,16,19,20,32,33}$ Such genetic variations, referred to as "functional polymorphisms" because of their association with clinical phenotypes, 4,27 include the eNOS gene intron 4 27-base pair VNTR variant (designated eNOS 27 VNTR variant); 32,33 the $e N O S$ gene promoter SNP (eNOS T-786C SNP); ${ }^{16,22}$ and the eNOS gene exon 7 SNP (eNOS G894T SNP). ${ }^{7.8}$ The polymorphisms we studied are depicted in Fig. 1.

\section{New Genetic Evidence for Susceptibility to Aneurysm Rupture}

The $e$ NOS Gene as a Marker for Rupture Susceptibility. We recently reported the clinical and genetic data obtained in 51 patients with radiologically proven aneurysmal SAH and 90 volunteers selected randomly from a community-based registry. ${ }^{15}$ In that study, by using multiple logistic regression analysis and adjusting for age, sex, and smoking history, we found a significant difference in the distribution of genotypes for the eNOS 27 VNTR polymorphism in patients with SAH compared with the volunteer (control) group $(p=0.002)$. Heterozygosity for this polymorphism was almost three times as prevalent among patients with $\mathrm{SAH}$ as in controls $(\mathrm{p}=$ 0.002 ). The "4a" allele (that is, the putatively variant or abnormal allele) was found in $51 \%$ of patients, compared with $22 \%$ of controls; this difference was significant $(\mathrm{p}=$ 0.007). In comparisons of patients with SAH with controls, the odds ratio for harboring an aneurysm in those 


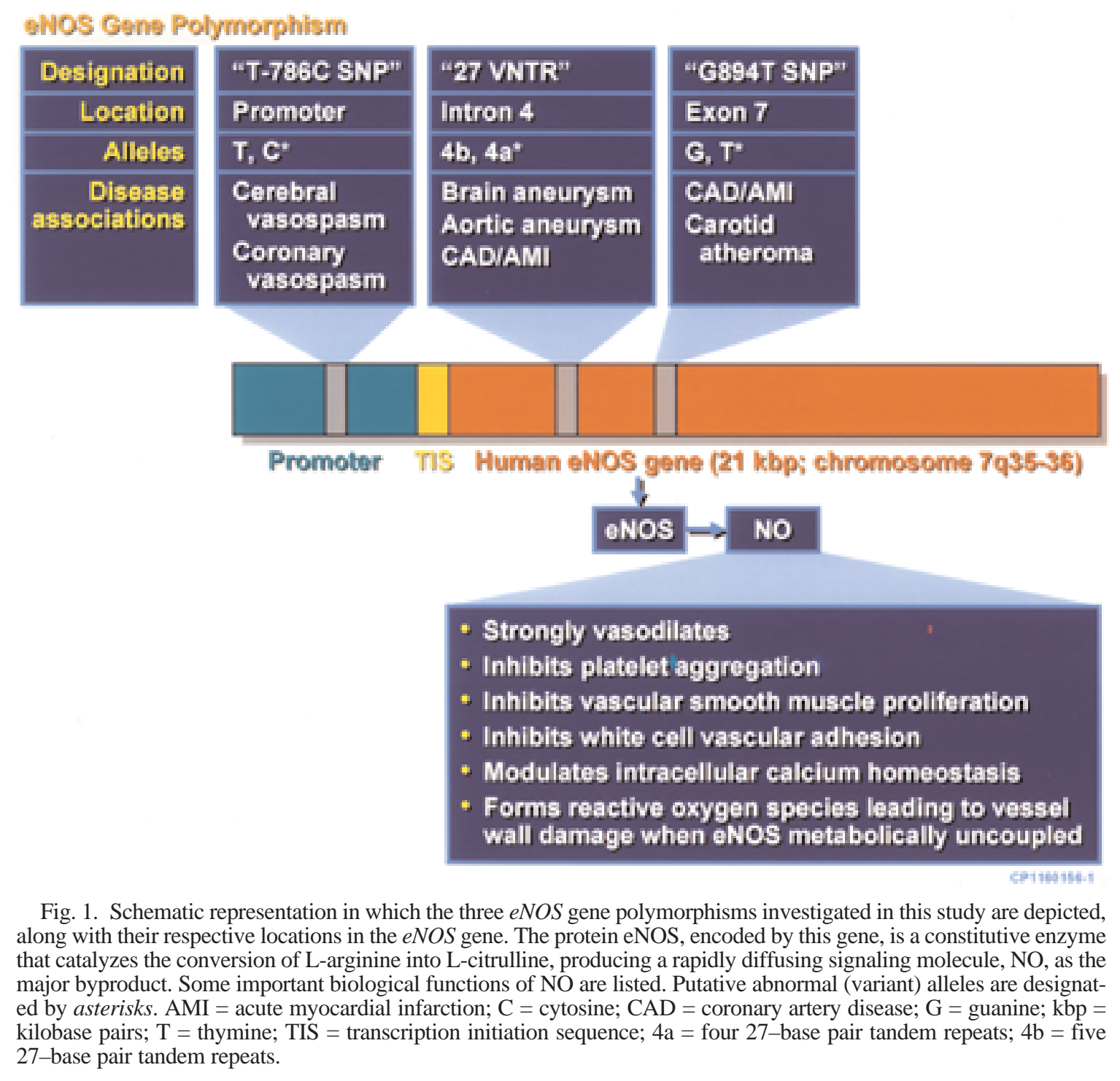

with at least one $4 \mathrm{a}$ allele was 3.95 (95\% confidence interval $1.45-10.65 ; p=0.007$ ) after controlling for differences among patients with regard to age, smoking status, and sex.

Our finding with regard to the eNOS 27 VNTR variant and increased susceptibility to intracranial aneurysm rupture supports the work of Wang, et al., ${ }^{32}$ and Kotani, et al. ${ }^{17}$ who found that the 4 a allele of this polymorphism was associated with increased susceptibility to cardiovascular disease; specifically coronary artery disease and aortic aneurysm rupture. Although the rarity of the $4 \mathrm{a} / 4 \mathrm{a}$ homozygote in participants in our study precluded statistical comparisons of this genotype between patients and control volunteers, we found that heterozygosity (involving carriage of the putative abnormal $4 \mathrm{a}$ allele) occurred almost three times as frequently in patients with SAH as in controls. It is important to note that polymorphic NOS dysfunction may result from the transcription and translation of a putatively abnormal allele in either the homozygous or heterozygous form. ${ }^{22}$ The precise molecular effects of these polymorphisms have not been elucidat- ed, although there is biochemical evidence for decreased eNOS gene promoter activation associated with the T786C SNP variant and reduced eNOS protein expression and enzymatic activity associated with both the eNOS 27 VNTR and T-786C SNP variants. ${ }^{22,30}$ It is certainly conceivable that such variants may contribute to aneurysm pathobiology and cerebral vasospasm through increased local oxidative stress leading to vessel wall damage, predilection toward development of atherogenic intimal hyperplasia, and systemic hypertension; the presence of aberrant vascular smooth-muscle cell proliferation; and increased platelet aggregation and proinflammatory monocyte adhesion, all of which are associated with NO signaling dysfunction. $3,6,11,12,18,21$

\section{Multiple Variant eNOS Alleles Strongly Associated With Aneurysm Rupture}

The notion of rupture-prone and rupture-resistant subpopulations of brain aneurysms is further reinforced by the novel genetic data reported in our most recent study 
(VG Khurana, et al., 2004, manuscript in review). In that study, despite the similarities in demographic and clinical characteristics between our groups with ruptured (58 patients) and unruptured (49 patients) intracranial saccular aneurysms, the genetic differences between the two groups were striking. We found that polymorphic variant eNOS alleles (Fig. 1) and their corresponding genotypes were between two and four times more frequent among patients with SAH than in those with unruptured lesions, and our haplotype analysis indicated that the presence of two or three variant alleles was associated with an 8.6 to 11.4 increase in the odds of presenting with a ruptured brain aneurysm $(\mathrm{p}<0.01 ; 95 \%$ confidence interval $1.7-$ 75.9). Taken together, our anatomical and genetic data indicate that there are distinct differences between ruptured and unruptured aneurysms: the former tend to be smaller, have a greater predilection for the anterior and posterior communicating arteries, and have a tendency to occur more commonly in persons with two or three variant eNOS polymorphic alleles.

\section{Clinical Implications of These Data}

Among the estimated 5 to $15 \%$ of aneurysm-harboring individuals with a relatively strong family history of brain aneurysms or with a heritable connective tissue disorder (such as Ehlers-Danlos or Marfan syndromes, or autosomal-dominant polycystic kidney disease), ${ }^{29}$ noninvasive radiological screening for brain aneurysms is accepted as being worthwhile. ${ }^{1}$ Nevertheless, for the remaining majority of persons at this time classified as having sporadic unruptured brain aneurysms, there is currently no adequate screening tool. To identify such individuals through population-wide serial radiological screening seems largely impractical, and no "aneurysm gene" has yet been identified. Therefore, perhaps one of the most important aspects of current management practices relates to how to counsel a patient with a newly diagnosed brain aneurysm, that is, regarding observation compared with treatment.

Findings in the ISUIA have indicated that certain aneurysms are more prone to rupture; however, for reasons given earlier and elaborated in detail elsewhere, ${ }^{5,16}$ advice based on the ISUIA data alone may not cover the gamut of rupture-prone aneurysms. We therefore believe that the findings in our recent genetic studies shed new and important light on the genetic basis of what are otherwise identified as sporadic brain aneurysms (found in $85-95 \%$ of all aneurysm-harboring individuals). For example, a person who receives a diagnosis, incidental or otherwise, of an unruptured intracranial aneurysm (especially one located in a higher-risk cerebrovascular territory) and in whom two or three variant eNOS polymorphic alleles (Fig. 1) are found with gene microarray technology (now becoming more readily available), would be counseled toward earlier treatment rather than observation based on the data reported herein.

\section{CONCLUSIONS}

We believe that if our findings are reproducible in the setting of a large multicenter study, then in addition to known anatomical factors, a rapid and cost-effective screening tool will become available to clinicians as a genetic aid for predicting rupture risks in patients presenting with unruptured intracranial aneurysms.

\section{Acknowledgment}

We thank Ms. Mary Soper for assistance with preparation of this article.

\section{References}

1. Bederson JB, Awad IA, Wiebers DO, Piepgras D, Haley EC Jr, Brott $\mathrm{T}$, et al: Recommendations for the management of patients with unruptured intracranial aneurysms: a statement for healthcare professionals from the Stroke Council of the American Heart Association. Stroke 31:2742-2750, 2000

2. Dalkara T, Moskowitz MA: Nitric oxide and the cerebral circulation, in Welch KMA, Caplan LR, Reis DJ, Siesjo BK, Weir B (eds): Primer on Cerebrovascular Diseases. San Diego: Academic Press, 1997, pp 96-98

3. Dumont AS, Dumont RJ, Chow MM, Lin CL, Calisaneller T, Ley KF, et al: Cerebral vasospasm after subarachnoid hemorrhage: putative role of inflammation. Neurosurgery 53: 123-135, 2003

4. Evans WE, Relling MV: Pharmacogenomics: translating functional genomics into rational therapeutics. Science 286: 487-491, 1999

5. Forget TR Jr, Benitez R, Veznedaroglu E, Sharan A, Mitchell W, Silva M, et al: A review of size and location of ruptured intracranial aneurysms. Neurosurgery 49:1322-1326, 2001

6. Guzik TJ, West NE, Pillai R, Taggart DP, Channon KM: Nitric oxide modulates superoxide release and peroxynitrite formation in human blood vessels. Hypertension 39:1088-1094, 2002

7. Hingorani AD: Polymorphisms in endothelial nitric oxide synthase and atherogenesis: John French Lecture 2000. Atherosclerosis 154:521-527, 2000

8. Hingorani AD, Liang CF, Fatibene J, Lyon A, Monteith S, Parsons A, et al: A common variant of the endothelial nitric oxide synthase $\left(\mathrm{Glu}^{298} \rightarrow \mathrm{Asp}\right)$ is a major risk factor for coronary artery disease in the UK. Circulation 100:1515-1520, 1999

9. Inagawa T, Hirano A: Autopsy study of unruptured incidental intracranial aneurysms. Surg Neurol 34:361-365, 1990

10. International Study of Unruptured Intracranial Aneurysm Investigators: Unruptured intracranial aneurysms-risk of rupture and risks of surgical intervention. N Engl J Med 339: 1725-1733, 1998

11. Johanning JM, Armstrong PJ, Franklin DP, Han DC, Carey DJ, Elmore JR: Nitric oxide in experimental aneurysm formation: early events and consequences of nitric oxide inhibition. Ann Vasc Surg 16:65-72, 2002

12. Khurana VG, Besser M: The pathophysiological basis of cerebral vasospasm following aneurysmal subarachnoid haemorrhage. J Clin Neurosci 4:122-131, 1997

13. Khurana VG, Smith LA, Baker TA, Eguchi D, O'Brien T, Katusic ZS: Protective vasomotor effects of in vivo recombinant endothelial nitric oxide synthase gene expression in a canine model of cerebral vasospasm. Stroke 33:782-789, 2002

14. Khurana VG, Smith LA, Weiler DA, Springett MJ, Parisi JE, Meyer FB, et al: Adenovirus-mediated gene transfer to human cerebral arteries. J Cereb Blood Flow Metab 20:1360-1371, 2000

15. Khurana VG, Sohni YR, Mangrum WI, McClelland RL, O'Kane DJ, Meyer FB, et al: Endothelial nitric oxide synthase gene polymorphisms predict susceptibility to aneurysmal subarachnoid hemorrhage and cerebral vasospasm. J Cereb Blood Flow Metab 24:291-297, 2004

16. Khurana VG, Sohni YR, Mangrum WI, McClelland RL, O'Kane DJ, Meyer FB, et al: Endothelial nitric oxide synthase T-786C single nucleotide polymorphism: a putative genetic marker differentiating small versus large ruptured intracranial aneurysms. Stroke 34:2555-2559, 2003 
17. Kotani K, Shimomura T, Murakami F, Ikawa S, Kanaoka Y, Ohgi S, et al: Allele frequency of human endothelial nitric oxide synthase gene polymorphism in abdominal aortic aneurysm. Intern Med 39:537-539, 2000

18. Kuhlencordt PJ, Gyurko R, Han F, Scherrer-Crosbie M, Aretz TH, Hajjar R, et al: Accelerated atherosclerosis, aortic aneurysm formation, and ischemic heart disease in apolipoprotein E/endothelial nitric oxide synthase double-knockout mice. Circulation 104:448-454, 2001

19. Lembo G, De Luca N, Battagli C, Iovino G, Aretini A, Musicco $\mathrm{M}$, et al: A common variant of endothelial nitric oxide synthase (Glu298Asp) is an independent risk factor for carotid atherosclerosis. Stroke 32:735-740, 2001

20. Marsden PA, Heng HH, Scherer SW, Stewart RJ, Hall AV, Shi $\mathrm{XM}$, et al: Structure and chromosomal localization of the human constitutive endothelial nitric oxide synthase gene. J Biol Chem 268:17478-17488, 1993

21. Moncada S, Palmer RM, Higgs EA: Nitric oxide: physiology, pathophysiology, and pharmacology. Pharmacol Rev 43: 109-142, 1991

22. Nakayama M, Yasue H, Yoshimura M, Shimasaki Y, Kugiyama $\mathrm{K}$, Ogawa $\mathrm{H}$, et al: $\mathrm{T}-786 \rightarrow \mathrm{C}$ mutation in the $5^{\prime}$-flanking region of the endothelial nitric oxide synthase gene is associated with coronary spasm. Circulation 99:2864-2870, 1999

23. Onda H, Kasuya H, Yoneyama T, Takakura K, Hori T, Takeda $\mathrm{J}$, et al: Genomewide-linkage and haplotype-association studies map intracranial aneurysm to chromosome 7q11. Am J Hum Genet 69:804-819, 2001

24. Peters DG, Kassam A, St Jean PL, Yonas H, Ferrell RE: Functional polymorphism in the matrix metalloproteinase-9 promoter as a potential risk factor for intracranial aneurysm. Stroke 30:2612-2616, 1999

25. Roses AD: Genetic susceptibility to cardiovascular diseases. Am Heart J 140:S45-S47, 2000

26. Rubin EM, Tall A: Perspectives for vascular genomics. Nature 407:265-269, 2000

27. Rusnak JM, Kisabeth RM, Herbert DP, McNeil DM: Pharmacogenomics: a clinician's primer on emerging technologies for improved patient care. Mayo Clin Proc 76:299-309, 2001
28. Sadamitsu D, Kuroda Y, Nagamitsu T, Tsuruta R, Inoue T, Ueda T, et al: Cerebrospinal fluid and plasma concentrations of nitric oxide metabolites in postoperative patients with subarachnoid hemorrhage. Crit Care Med 29:77-79, 2001

29. Schievink WI: Intracranial aneurysms. N Engl J Med 336: 28-40, 1997

30. Song J, Yoon Y, Park KU, Park J, Hong YJ, Hong SH, et al: Genotype-specific influence on nitric oxide synthase gene expression, protein concentrations, and enzyme activity in cultured human endothelial cells. Clin Chem 49:847-852, 2003

31. Testa C, Andreoli A, Arista A, Limoni P, Tognetti F: Overall results in 304 consecutive patients with acute spontaneous subarachnoid hemorrhage. Surg Neurol 24:377-385, 1985

32. Wang XL, Sim AS, Badenhop RF, McCredie RM, Wilcken DE: A smoking-dependent risk of coronary artery disease associated with a polymorphism of the endothelial nitric oxide synthase gene. Nat Med 2:41-45, 1996

33. Wang XL, Wang J: Endothelial nitric oxide synthase gene sequence variations and vascular disease. Mol Genet Metab 70: 241-251, 2000

34. Weir B, Macdonald L: Cerebral vasospasm. Clin Neurosurg 40:40-55, 1993

35. Wiebers DO, Whisnant JP, O'Fallon WM: The natural history of unruptured intracranial aneurysms. N Engl J Med 304: 696-698, 1981

36. Wiebers DO, Whisnant JP, Sundt TM Jr, O'Fallon WM: The significance of unruptured intracranial saccular aneurysms. J Neurosurg 66:23-29, 1987

Manuscript received September 15, 2004.

Accepted in final form October 5, 2004.

Our genomics studies were funded by the Departments of Neurologic Surgery and Neurology, Mayo Clinic and Mayo Foundation, Rochester, Minnesota.

Address reprint requests to: Vini G. Khurana, M.D., Ph.D., Department of Neurologic Surgery, Mayo Clinic, 200 First Street SW, Rochester, Minnesota 55905. email: khurana.gautam@mayo.edu. 